\title{
Modeling and Compensation Method for Temperature Error of FOG based on the SVM
}

\author{
Guochen Wang * \\ Collage of Automation \\ Harbin Engineering University \\ Harbin, China \\ E-mail: akmango@126.com \\ * Corresponding Author \\ Wei Gao \\ Collage of Automation \\ Harbin Engineering University \\ Harbin, China
}

\author{
Bo Zhao \\ Anshan Industrial Technology Research Institute of \\ Harbin Institute of Technology \\ Anshan, China
}

\author{
Runfeng Zhang \\ Anshan Industrial Technology Research Institute of \\ Harbin Institute of Technology \\ Anshan, China
}

\begin{abstract}
The performance of the Fiber Optical Gyroscope (FOG) is susceptible to the external environment temperature, and the temperature effect of the FOG has nonlinear characteristics. Therefore, it is necessary to develop an outstanding temperature compensation method with high precision and good versatility. In this paper, the severe influence of environmental temperature on the FOG's accuracy was analyzed in detailed. Then the temperature error model of the FOG was established. Based on the function fitting capability of the Support Vector Machine (SVM), a new modeling and compensation method of the gyroscope's temperature error was proposed. To verify the advantages of this novel method, we compared it with other two compensation methods, which are based on the classical statistical estimation parameters (polynomial regression) and the neural network, respectively. Experiment results showed that the proposed method can decrease the temperature error of the FOG by $75 \%$ and improve the adaptability of the environmental temperature. With this novel method, the gyro bias stability can meet the practical need of the temperature compensation which requires highprecision FOG.
\end{abstract}

Keywords-Component; Fiber Optical Gyroscope (FOG); Temperature Error; Support Vector Machine (SVM); Bias Stability; Error Compensation

\section{INTRODUCTION}

The Fiber Optical Gy roscope (FOG) is an angular motion measurement instrument based on the Sagnac principle [1]. Due to its characteristics of all solid, longtime service, fast startup and widely measuring dynamic range etc., it has been the dominant measurement device of the new generation inertial guidance and measurement system. As the core components of the FOG are sensitive to the temperature, the self-heating of the FOG and the environmental temperature change become a big challenge of the FOG in engineering fields [2].

To solve this problem, the most commonly used methods are the temperature control and the temperature compensation methods in the practical engineering. The temperature control technology is commonly used to ensure the environment temperature stability when the FOG is working. However, using this method, it do not only need additional temperature controllers in gyroscope but also require high performance of the controllers, which will increase the FOG's size, weight and cost inevitably and the accuracy of the temperature control is also restricted [3]. In addition, the compensation method of the temperature error using the software is a pure mathematics method. In this method, the temperature characteristics of the FOG are described accurately to improve the gyro's detection accuracy, which is crucial for the FOG to be applied in practical applications. In [4] the FOG's bias variation with temperature was approximately linearized to obtain a linear model of the gyro's temperature drift; Reference [5] applied the mixed linear regression model which considered the temperature and the temperature gradient to establish the temperature model of the FOG's drift ; [6] proposed a joint multi-parametric linear model, in which a simplified FOG equivalent phase model combines with the temperature sensitive parameter model to obtain the distribution model of the FOG's temperature drift; [7] adopted the BP neural network and the mathematical statistical methods to establish the gyro temperature compensation model; [8] used the wavelet network to identify the nonlinear temperature model of FOG; [9] applied the above fuzzy logic to achieve the model identification of the FOG's temperature drift and the self-compensation scheme. However, the FOG's temperature error has nonlinear characteristics. Therefore, it will produce errors just using linear regression model, and thus it is difficult to meet the high-precision demands. What's more, with the neural network modeling, the convergence rate is slow and there are some fatal shortcomings, such as easy to fall into local minima and poor generalization capability. All of these methods cannot meet the require ments of the FOG's PSO temperature error compensation. 
The support vector machine (SVM) is a new machine learning method, which can be applied into the pattern recognition, function approximation and some other areas [10-11]. It can effectively solve the small sample, nonlinearity, high dimension and local minima problems, with high accuracy, strong generalization ability and other characteristics [12]. After analyzed the FOG's temperature error model, a new FOG temperature error modeling and compensation method was proposed in this paper, combined with the SVM function fitting function. And some experiments were carried out to verify the effectiveness of this novelmethod.

\section{TEMPERATURE ERROR OF THE FOG}

\section{A. Analysis of the FOG's Temperature Error}

The effects of temperature on FOG include both noise and drift. In [11], the effects were deduced when two beam interference light transmit along the clockwise and counterclockwise, respectively. The phase delay of the thermotropic nonreciprocal in the fiber loop caused by temperature changes can be described as follows:

$$
\Delta \Phi_{e}=\frac{\beta_{o} n}{c_{o}} \frac{\partial n}{\partial T} \int_{o}^{L}[\Delta T(z)-\Delta T(L-z)](2 z-L) d z
$$

where $\beta_{o}=2 \pi / \lambda_{0}$ is the propagation constant of the light in vacuum; $c_{o}$ represents the light speed in the waveguide; $\Delta \mathrm{T}(\mathrm{z})$ represents the temperature change in the $\mathrm{z}$ axis of the fiber; $L$ is the total length of the fiber; $n$ represents the fiber's refractive index. It is can be seen from (1) that the size of the non-reciprocity is affected not only by the distribution and the change of the environmental temperature, but also by the parameters and the winding method of the fiber loop. Thus the compensation method of the FOG's temperature drift error includes four as pects: improvements of the FOG's structure and components, development of the fiber loop winding technology, the temperature control of the FOG and the error modeling and compensation.

\section{B. Modeling and Compensation of the Temperature Drift}

Generally speaking, the FOG is a kind of the optical gyroscope based on the Sagnac principle. Its input-output model can be simply described as Fig. 1:

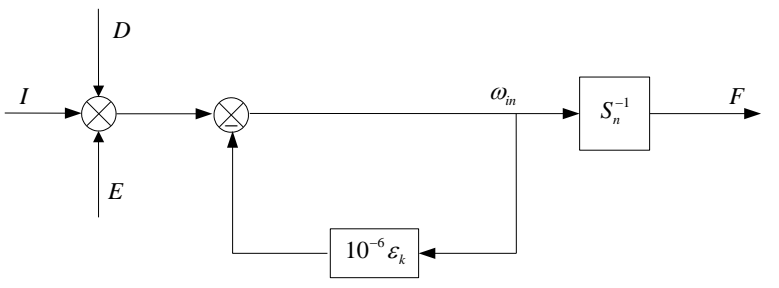

Figure 1. Input-output model of the FOG.

The measurement model equation can be expressed as:

$$
S_{0}(F)=\frac{I+E+D}{1+10^{-6} \varepsilon_{k}}
$$

Where, $S_{0}$ represents the nominal scale factor, $F$ is the output rate, $I$ represents the input angular velocity, $E$ is the temperature error, $D$ represents the drift error, and $\varepsilon_{K}$ represents the scale factor error. Further, the temperature error of the FOG can be approximated as follows:

$$
E=D_{T} \Delta T+D_{\nabla_{T}} \nabla T+D_{\dot{T}} \dot{T}
$$

where $\Delta T$ represents the gyro's temperature change, $D_{T}$ represents error temperature variable coefficient, $T$ represents the temperature gradient of the gyro, $D_{\nabla_{T}}$ represents error gradient coefficient, $\dot{T}$ represents gyro temperature change rate, $D_{\dot{T}}$ represents error rate coefficient.

From (3), it can be seen that the FOG's temperature error is mainly caused by the temperature variation, the temperature gradient and the temperature change rate. And to some extent, the error is linear approximately. However, in practice, when the environmental temperature changes drastically or the gyro components heat, which can promote the formation of the non-equilibrium temperature field inside the gyro, $\Delta T 、 \nabla T$ and $\dot{T}$ will be difficult to be obtained.

Take a certain type of the gyro as an example, 3 platinum resistances used for temperature measuring are set in the anode, the cathode of the gyro's cavity and the inner surface of the gyro's cartridge, respectively. The output of the temperature data are $T_{a} 、 T_{b}$ and $T_{c}$, the approximately linear relationship can be described as (4).

$$
\left\{\begin{array}{l}
\Delta T=m_{0}+m_{1}\left(T_{a}-T_{a 0}\right)+m_{2}\left(T_{b}-T_{b 0}\right)+m_{3}\left(T_{c}-T_{c 0}\right) \\
\nabla T=n_{0}+n_{1}\left(T_{a}-T_{b}\right)+n_{2}\left(T_{b}-T_{c}\right) \\
\dot{T}=l_{0}+l_{1} \dot{T}_{a}+l_{2} \dot{T}_{b}+l_{3} \dot{T}_{c}
\end{array}\right.
$$

Where, $T_{a 0}, T_{b 0}$ and $T_{c 0}$ represent the reference temperature of the measurement points; $\dot{T}_{a}, \dot{T}_{b}$ and $\dot{T}_{c}$ represent the temperature rate of the measurement point; and $m_{i}(i=0,1, \cdots, 3), n_{j}(j=0,1,2)$ and $l_{k}(k=0,1, \cdots, 3)$ are the corres ponding coefficients.

Using the FOG's temperature model described by (3) and (4), However, the problem of the linear quadratic approximation is existed which will limit the accuracy of the temperature error compensation inevitably. And furthermore, the measuring accuracy of the gyro will be affected. Since the temperature error of the FOG is decided by its internal temperature field, so a nonlinear model between the temperature sampling and the temperature error can be directly established, and thereby the accuracy of the temperature error compensation can be improved availably.

\section{MODELING AND COMPENSATION OF THE FOG'S TEMPERATURE ERROR BASED ON THE SVM}

\section{A. The Function Fitting Method of the SVM}

The core of the function fitting problem is to determine the unknown function $y=f(x)$, which ensure that the distance between the estimation function $\hat{f}(x)$ and $f(x)$ is the minimum, and the distance can be described by (5).

$$
R(f, \hat{f})=\int L(f, \hat{f}) \mathrm{d} x
$$


Where, $L$ represents the loss function. As the function $f(x)$ is unknown, $\hat{f}(x)$ can only be solved by the sample data $\left(x_{1}, y_{1}\right),\left(x_{2}, y_{2}\right), \ldots,\left(x_{k}, y_{k}\right)$.

In the SVM, Equation (6) as below can be adopted to approximately fit the unknown function:

$$
y=f(x)=w^{T} \varphi(x)+b
$$

Where $x \in R^{n}, y \in R$ represent the independent variable and the dependent variable of the fitting function, respectively; $\varphi(\cdot)$ represents the feature function which enables the independent variable to map into the highdimensional space. And in the above equation the nonlinear function in the low-dimensional space is mapped into the high-dimensional space and then fitted linearly. Assume that all training data can be fitted the linear function under the accuracy of $\varepsilon$ without any error, that is:

$$
\left\{\begin{array}{l}
y_{i}-\left\langle w, x_{i}\right\rangle-b \leq \varepsilon \\
\left\langle w, x_{i}\right\rangle+b-y_{i} \leq \varepsilon
\end{array} \quad i=1,2, \cdots, k\right.
$$

Considering that the fitting error is allowed, the relaxation factors $\xi_{i}^{*} \geq 0$ and $\xi_{i} \geq 0$ are introduced, so (7) can be rewritten as below:

$$
\left\{\begin{array}{l}
y_{i}-\left\langle w, x_{i}\right\rangle-b \leq \varepsilon+\xi_{i} \\
\left\langle w, x_{i}\right\rangle+b-y_{i} \leq \varepsilon+\xi_{i}^{*} \quad i=1,2, \cdots, k \\
\xi_{i}, \xi_{i}^{*} \geq 0
\end{array}\right.
$$

According to the constraints of (8), the function fitting problem can be changed into a minimization problem of a function described by (9):

$$
R\left(w, \xi_{i}, \xi_{i}^{*}\right)=\frac{1}{2}\|w\|^{2}+C \sum_{i=1}^{k}\left(\xi_{i}+\xi_{i}^{*}\right)
$$

Where, the first term on the right side $\frac{1}{2}\|w\|^{2}$ can make the regression function much steadier, and thus the generalization ability can be improved effectively. And the second term can reduce the fitting error which usually adopts the insensitive penalty function. $\varepsilon$ is a positive constant, and the difference between $f\left(x_{i}\right)$ and $y_{i}$ will not be counted into the error when the difference is less than $\varepsilon$ :

$$
\left|f\left(x_{i}\right)-y_{i}\right|= \begin{cases}0 & \left|f\left(x_{i}\right)-y_{i}\right|<\varepsilon \\ \left|f\left(x_{i}\right)-y_{i}\right|-\varepsilon & \left|f\left(x_{i}\right)-y_{i}\right| \geq \varepsilon\end{cases}
$$

The constant $C>0$ is the penalty factor and it can control the importance degree of the samples which beyond the error $\varepsilon$.

The minimization of (9) is a convex quadratic optimization problem, and the Lagrangian function is introduced:

$$
\begin{aligned}
& L\left(w, b, \xi, \xi^{*}, a, a^{*}, \gamma, \gamma^{*}\right)=\frac{1}{2}\|w\|^{2}+ \\
& C \sum_{i=1}^{k}\left(\xi_{i}+\xi_{i}^{*}\right)-\sum_{i=1}^{k} a_{i}\left|\xi_{i}+\varepsilon-y_{i}+f\left(x_{i}\right)\right|- \\
& \sum_{i=1}^{k} a_{i}\left|\xi_{i}+\varepsilon-y_{i}+f\left(\boldsymbol{x}_{i}\right)\right|-\sum_{i=1}^{k}\left(\xi_{i} \gamma_{i}+\xi_{i}^{*} \gamma_{i}^{*}\right)
\end{aligned}
$$

Where, $a, a^{*} \geq 0, \gamma, \gamma^{*} \geq 0, \quad i=1,2, \cdots, k$. The optimal solution of (9) is the saddle point of (11). At the saddle point, the function $L$ can get the minimum on $w, b, \xi, \xi^{*}$ and the maximum on $a, a^{*}, \gamma, \gamma^{*}$. Therefore, the minimization problem of (9) can be converted to seek the maximization problem of the dual problem, and the dual problem can be expressed as:

$$
\hat{w}\left(a, a^{*}, \gamma, \gamma^{*}\right)=\min _{w, b, \xi, \xi^{*}} L^{\left(w, b, \xi, \xi^{*}\right)}
$$

At the saddle point, the Lagrangian function $L$ can get the minimum point on $w, b, \xi, \xi^{*}$, and we can also obtain this:

$$
\left\{\begin{array}{l}
\frac{\partial}{\partial_{w}} L=0 \Rightarrow \boldsymbol{w}-\sum_{i=1}^{k}\left(a_{i}-a_{i}^{*}\right) x_{i}=0 \\
\frac{\partial}{\partial_{b}} L=0 \Rightarrow \sum_{i=1}^{k}\left(a_{i}-a_{i}^{*}\right)=0 \\
\frac{\partial}{\partial_{\xi_{i}}} L=0 \Rightarrow C-a_{i}-\gamma_{i}=0 \\
\frac{\partial}{\partial_{\xi_{i}^{*}}} L=0 \Rightarrow C-a_{i}^{*}-\gamma_{i}^{*}=0
\end{array}\right.
$$

Substituting (13) into (11), the dual function of the Lagrangian function can be obtained by (14):

$$
\begin{aligned}
& \max \boldsymbol{w}\left(a, a^{*}\right)=-\frac{1}{2} \sum_{i, j=1}^{k}\left(a_{i}-a_{i}^{*}\right)\left(a_{j}-a_{j}^{*}\right)\left\langle\boldsymbol{x}_{i}, \boldsymbol{x}_{j}\right\rangle- \\
& \sum_{i=1}^{k}\left(a_{i}+a_{i}^{*}\right) \varepsilon+\sum_{i=1}^{k}\left(a_{i}-a_{i}^{*}\right) y_{i} \\
& \text { s.t. }\left\{\begin{array}{l}
\sum_{i=1}^{k}\left(a_{i}-a_{i}^{*}\right)=0 \\
0 \leq a_{i}, a_{i}^{*} \leq C, i=1,2, \cdots, k
\end{array}\right.
\end{aligned}
$$

For the nonlinear regression, firstly, the data should be mapped to a high dimensional feature space using a nonlinear mapping function $x \rightarrow \varphi(x)$, and then the linear regression can be realized in this space. After that, we can obtain the nonlinear regression effects in the original space. Meanwhile, introducing the kernel function $k\left(\boldsymbol{x}_{\boldsymbol{i}}, \boldsymbol{x}_{\boldsymbol{j}}\right)=\left\langle\varphi\left(\boldsymbol{x}_{\boldsymbol{i}}\right), \varphi\left(\boldsymbol{x}_{\boldsymbol{j}}\right)\right\rangle$ that complies with the Mercer condition, the following will be got:

$$
\begin{aligned}
& \max \boldsymbol{w}\left(a, a^{*}\right)=-\frac{1}{2} \sum_{i, j=1}^{k}\left(a_{i}-a_{i}^{*}\right)\left(a_{j}-a_{j}^{*}\right) k\left(\boldsymbol{x}_{\boldsymbol{i}}, \boldsymbol{x}_{\boldsymbol{j}}\right)- \\
& \sum_{i=1}^{k}\left(a_{i}+a_{i}^{*}\right) \varepsilon+\sum_{i=1}^{k}\left(a_{i}-a_{i}^{*}\right) y_{i} \\
& \text { s.t. }\left\{\begin{array}{l}
\sum_{i=1}^{k}\left(a_{i}-a_{i}^{*}\right)=0 \\
0 \leq a_{i}, a_{i}^{*} \leq C, i=1,2, \cdots, k
\end{array}\right.
\end{aligned}
$$

Then,

$$
w=\sum_{i=1}^{k}\left(a_{i}-a_{i}^{*}\right) \varphi\left(\boldsymbol{x}_{\boldsymbol{i}}\right)
$$

When $\langle w, x\rangle=w_{0}$, the regression function $f(x)$ can be obtained as: 


$$
f(\boldsymbol{x})=\sum_{i, j=1}^{k}\left(a_{i}-a_{i}^{*}\right) k\left(\boldsymbol{x}, \boldsymbol{x}_{\boldsymbol{j}}\right)+b=w_{0}+b
$$

Choosing a different kernel functions $k\left(\boldsymbol{x}, \boldsymbol{x}_{\boldsymbol{j}}\right)$, different SVMs can be formatted. In this paper, the radial basis function was selected as followed:

$$
k\left(\boldsymbol{x}, \boldsymbol{x}_{i}\right)=\exp \left(-\left\|\boldsymbol{x}-\boldsymbol{x}_{i}\right\|^{2} / 2 \sigma^{2}\right)
$$

Where, $\sigma^{2}$ is the kernel width of the radial basis function.

\section{B. Modeling of the FOG's Temperature Error Modeling}

According to the sample data of the temperature error by experiments, the FOG's temperature error is modeled by the fitting function method based on the SVM, including three steps: the data normalization, the parameter optimization and the model training.

\section{1) Data normalization}

To accelerate the parameter optimization, the model training and the optimization of the memory space, the following formula can be adopted to normalize the sample data.

$$
y=\frac{y_{\max }-y_{\min }}{x_{\max }-x_{\min }}\left(x-x_{\min }\right)+y_{\min }
$$

Where $y$ represents the normalized output and $y_{\max }=1, y_{\min }=-1 ; x$ represents the normalized input and $x_{\max }$ is the maximum of the input while $x_{\min }$ is the minimum. Arbitrary data in real number range can be compressed to the interval $[-1,1]$ by (19). The sampling data include the gyro temperature $\left(T_{a, i}, T_{b, i}, T_{c, i}\right)$ and the corresponding temperature error $E_{i}$, in which $i=1,2, \cdots, N$ and $N$ represents the sampling size. These four data are normalized and the corresponding models are recorded.

\section{2) Parameter optimization}

The parameters, which can affect the accuracy of the SVM function fitting directly, are mainly the penalty factor $\mathrm{C}$ and the RBF kernel width $\sigma^{2}$. In order to obtain the best fitting result, the grid optimization strategy was taken for the two key parameters. According to the experience, the value range of $\mathrm{C}$ and $\sigma^{2}$ is $\left[2^{-8}, 2^{8}\right]$. Taking 0.1 as a step, the values of $\mathrm{C}$ and $\sigma^{2}$ are in horizontal and vertical arrangement to construct the gridlike array, which is illustrated distinctly in Fig. 2. For the parameter of each grid cell, the corresponding fitting function model can be obtained. A set of parameters used to acquire the highest fitting accuracy are selected as the optimal parameters, and they are represented by $C_{\text {best }}$ and $\sigma_{\text {best }}^{2}$, respectively.

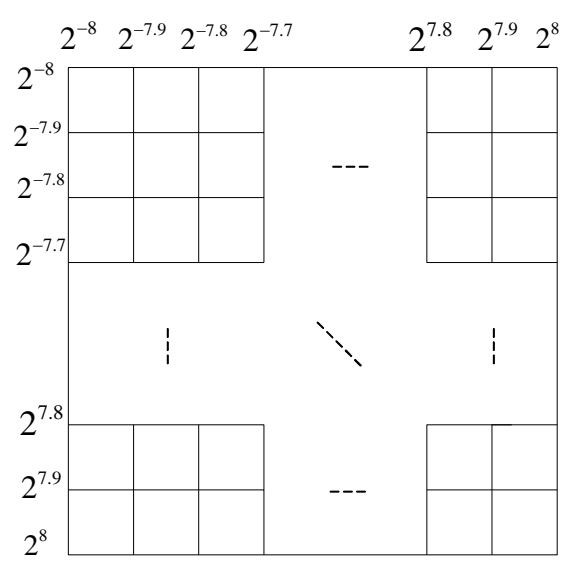

Figure 2. Grid used for optimal parameter searching.

Furthermore, to avoid the occurrence of the overlearning and less-learning states, the cross validation method is adopted when the grid optimization is executed. In the cross validation method, the original data are divided into $\mathrm{k}$ groups and each subset is taken as a validation set, and the other $K-1$ groups are regarded as the training set, and then $K$ models will be obtained. In the final validation set of the $K$ models, the average value of the fitting function's accuracy is used as the performance index for this classifier. In this paper, $K$ is set as 3 .

\section{3) Model training}

According to the SVM function fitting theory, after substituting the parameter optimization results and normalizing data to training, the best fitting function can be obtained as followed:

$$
f(\boldsymbol{y})=\left[\sum_{i=1}^{k}\left(a_{i}-a_{i}^{*}\right) \exp \left(-\left\|\boldsymbol{y}-\boldsymbol{y}_{k}\right\|^{2} / 2 \sigma^{2}\right)\right]+b
$$

Where $a_{i}$ and $a_{i}^{*}$ represents the corresponding Lagrange factors of the SVM, $b$ represents the bias term, and the temperature error model is expressed by (20) based on the SVM method.

\section{Temperature Error Compensation of the FOG}

According the above analysis, we proposed a novel compensation method of the temperature error here. The specific process of the proposed algorithm is as follows:

1) Normalize the gyro's original temperature data according to the established independent variable normalization model;

2) Substitute the normalized temperature data into the temperature error model to predict the error;

3) Reversely operate the calculation results according to the dependent variable normalization model to get the predicted temperature error of the FOG;

4) Finally, subtract the predicted temperature error in the original data from the gyro output to complete the temperature error compensation.

\section{EXPERIMENT RESULTS AND ANALYSIS}

\section{A. Calibration of the FOG's Temperature Error}

First, confirm that you have the correct template for $y$ Two single-axis FOGs (numbered \# 1 and \# 2) were selected whose nominal accuracy is better than $0.02^{\circ} / h$ 
when they were both fixed on the uniaxial rate turntable with a high-low temperature box and the turntable should be in an absolute state of rest. That is, the angular velocity of the gyro was the constant. In the gyro working process, the measurement data of the real-time angular velocity and 2-channel temperature data were output in the frequency of $100 \mathrm{~Hz}$.

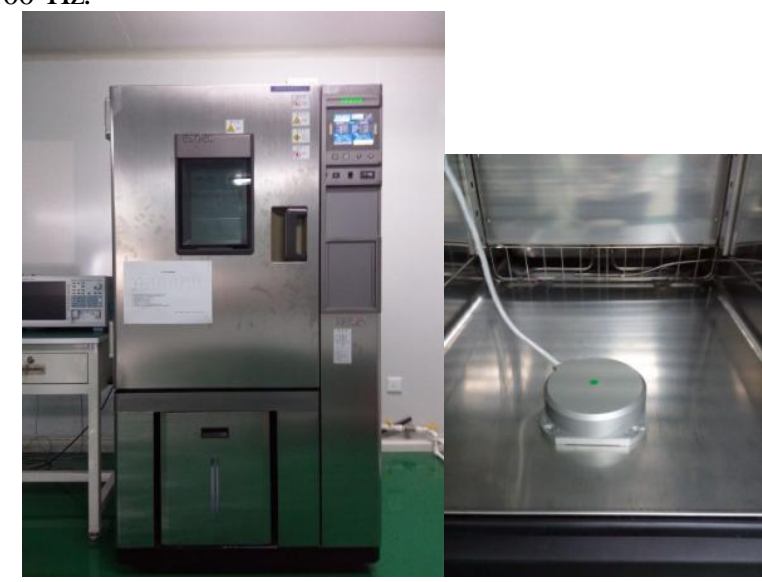

Figure 3. Curve of single-axis FOG in a temperature box.

Then a series of the experiments were done. First of all, the two gyros were energized and kept in the constant temperature of $18^{\circ} \mathrm{C}$ for $2.5 h$; Secondly, the temperature of the temperature box was set to fell to $-40^{\circ} \mathrm{C}$ at the rate of $-1^{\circ} \mathrm{C} / \mathrm{min}$ and kept for $2.5 h$; Thirdly, the temperature was set to rise to $60^{\circ} \mathrm{C}$ at the rate of $1^{\circ} \mathrm{C} / \mathrm{min}$ and kept for $2.5 h$. Subsequently, this kind of the temperature variation was executed 3 times.

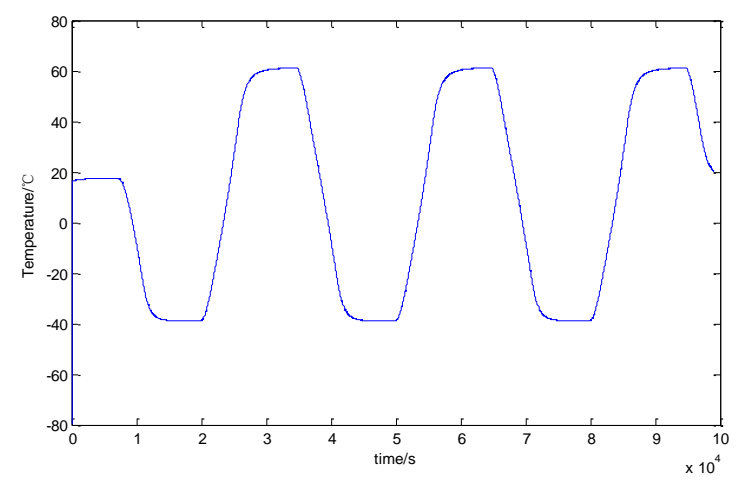

Figure 4. Temperature curve of thermal cabinet.

When the environment temperature is constant of $60^{\circ} \mathrm{C}$ and the inside of the FOG is in the thermal equilibrium, the average value of the gyro's output is regarded as the true measurement value, and in this experiment the temperature error was obtained from the collected data subtracted the true measurement. Meanwhile, since the FOG's output has a large quantization noise, so it cannot be directly used to calibrate the temperature error. Thus, the smoothed result of the data for 100s was used as the sampling point for the temperature error modeling. The te mperature data was also smoothed for $100 \mathrm{~s}$, and the smoothed curves were showed in Fig. 4. The curves of the Gyro's temperature error smoothed for 100s were showed in Fig. 5.

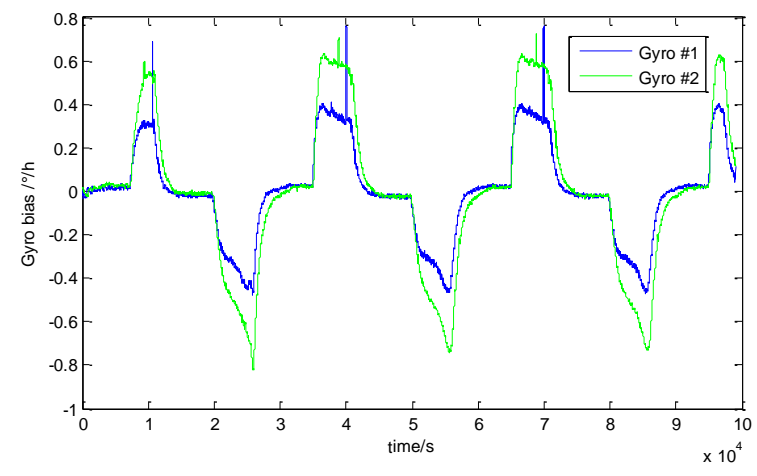

Figure 5. Gyro temperature error curves (without compensation).

As seen from Fig. 5, at the $2.5 \mathrm{~h}$ when the environment's temperature is constant $60{ }^{\circ} \mathrm{C}$, the error of the output temperature was close to 0 and its fluctuations of the standard deviation were $0.0125^{\circ} / h$ and $0.0153^{\circ} / h$, respectively, which are superior to nominal index $0.03^{\circ} / h$ of this gyro. However, during the whole experiment, the standard deviation of the gyro's output errors fluctuated with the temperature varying was about $0.3^{\circ} / h$, which is much larger than the nominal accuracy and the errors were magnified by 10 times.

The training sampling data collected in this experiment included $\left(T_{a, i}, T_{b, i}, \mathrm{~T}_{c, i}\right)$ and $E_{i}, i=1,2, \cdots, 336$. The temperature error calibration method proposed in chapter 3.2 was adopted to construct the gyro's temperature error model, and the main parameters of the model were showed in Table 1. Due to space reasons, the values of $a_{i}$ and $a_{i}^{*}$ were not listed in detail.

TABLE I. MAIN PARAMETERS OF GYRO TEMPERATURE ERROR MODEL

\begin{tabular}{cccc}
\hline Number & $C_{\text {best }}$ & $\sigma_{\text {best }}^{2}$ & $b$ \\
\hline$\# 1$ & 25.6534 & 0.3301 & 0.2794 \\
$\# 2$ & 83.4593 & 2.9786 & 0.6398 \\
\hline
\end{tabular}

\section{B. FOG's Temperature Error Compensation Results}

Although in the model calibration process the smoothed results for 100 s of the gyro's output data were used as the sample, the actual error prediction and compensation were the original output from the gyro at $100 \mathrm{~Hz}$. That is, the quantization noise of the gyro's measurement was not considered, and only the temperature error was eliminated.

Meanwhile, in order to verify the effectiveness of the FOG's temperature error modeling and compensation method based on the SVM, three methods, including the proposed method, the method based on the linear regression and the method based on the artificial neural network, were applied to compensate the FOG's high and low temperature experimental above. The comparis on of the temperature error curves before and after the compensation for \#1 and \#2 were showed in Fig. 6 and Fig. 7 , respectively. 


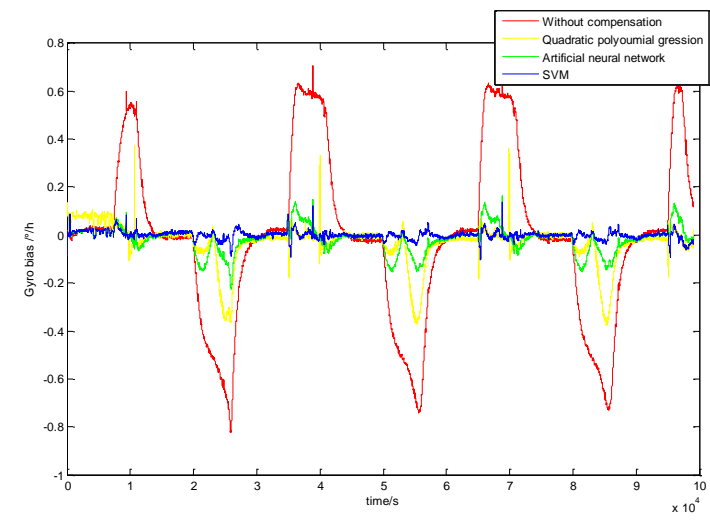

Figure 6. Comparison of bias error curves before and after compensation (\#1).

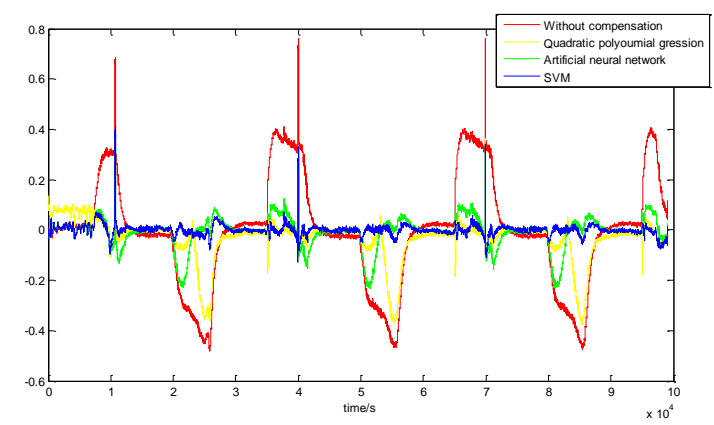

Figure 7. Comparison of bias error curves before and after compensation (\#2).

As showed in Fig. 5 and Fig. 6, the three methods were all effective for the FOG's temperature error compensation However, the stability of the data compensated by the proposed method in this paper was significantly higher than by the other two methods. After the further analysis of the standard deviation before and after the temperature compensation, the results were showed in Table 2. It can be seen that, using the linear regression based compensation method and the artificial neural network compensation method, the stability of the gyro's error can be improved, but it still cannot reach the nominal accuracy level. After adopting the SVM based compensation method proposed in this paper, the stability of the two gyros' error can reach the nominal accuracy level completely, and stability errors were reduced by about $94 \%$ and $86 \%$, respectively. Thus, with this method, the adaptability of the environmental temperature can be improved availably, and the feasibility and the superiority of this proposed method were also verified.

TABLE II. MODEL STANDARD DEVIATION OFTEMPERATURE ERROR BEFORE AND AFTER COMPENSATION $\left({ }^{\circ} / \mathrm{h}\right)$

\begin{tabular}{cccccc}
\hline \multicolumn{3}{c}{} & \multicolumn{4}{c}{ Compensation comparisons } \\
\cline { 3 - 6 } Num- & $\begin{array}{c}\text { Thermos- } \\
\text { ber }\end{array}$ & $\begin{array}{c}\text { Before } \\
\text { tatic } \\
\text { compe- } \\
\text { nsation }\end{array}$ & $\begin{array}{c}\text { Least } \\
\text { squares }\end{array}$ & $\begin{array}{c}\text { Artificial } \\
\text { neural } \\
\text { network }\end{array}$ & SVM \\
\hline \#1 & 0.0136 & 0.3087 & 0.0615 & 0.0548 & 0.0191 \\
$\# 2$ & 0.0164 & 0.1942 & 0.0877 & 0.0644 & 0.0279 \\
\hline
\end{tabular}

\section{CONCLUSIONS}

In this paper, considered the multi-parameter and nonlinear characteristics of the FOG's te mperature error model, a new error compensation method based on the SVM was proposed. In this method, the SVM was used to fit and approximate to the FOG's temperature error model, and based on this model the error can be predicted and compensated. The high and low temperature experiment results showed that the proposed method in this paper can not only effectively reduce the effects on the stability of the FOG's measurement caused by the environmental temperature, but also improve the compensation accuracy more significantly compared with the other two methods based on the linear regression and the artificial neural network. Therefore, the novel method proposed in this paper provided a new compensation way of the FOG's temperature error.

\section{REFERENCES}

[1] G. Pavlath. Challenges in the Development of the IFOG[C]. AIAA Guidance Navigation, and Control Conference and Exhibit, Austin, Texas. 2003: 11-14.

[2] Wang Hai. Effects of temperat ure and error compen sation for fiber optic gyro [J]. Journal of Beijing University of Aeronautics and Astronautics, 2007,33(5):549-551.

[3] Bai Junqing, ZhangKe, Geng Weimeng. The Research for FOG Inertial Navigation System of Modeling and Temperature Compensation Technology [J]. Piezoelectrics \& Acoustooptics, 2013, 35(2): 201- 203.

[4] Liu Guifu, Xing Yanli, Zhang Shuxia. Investigation on the Modeling of Fiber Optic Gyro Bias Instability [J]. Journal of Chinese Inertial Technology, 2001, 9(3): 48-52.

[5] Zhou Haibo, Liu Jianye, Lai Jizhou. Research on Mix Linear Regression Model of T emperat ure Compensation on FOG Drift [J]. The second session of the China Aviation Institute of Youth Science and Technology Forum Collection, 2006: 380-385.

[6] Jin Jing, Song Ningfang, Li Lijing. Temperature Drift Modeling and Real-time Compensation of Interferometric Fiber Optic Gyroscope [J]. Acta Aeronautica ET Astronautica Sinica ,2007, 28(6): 1449- 1454.

[7] Feng Lishuang, Nan Shuzhi, Jin Jing. Research on Modeling and Compensation Technology for Temperature Errors of FOG [J]. 2006, 27(5): 939-941, 1049.

[8] Cheng Yuming, Zhang Yanhua. Nonlinear Model Identification Of Fiber-Optic Gyroscope's Temperature Drif [J]. Journal of Shanghai Jiaotong University, 1997, 31(12): 123-125.

[9] ZHANG Hong-xian, WU Yan-ji, WANG Yu-Hui, BI Cong-Zhi. Temperature compensation for FOG based on fuzzy logic [J]. Journal of Chinese Inertial Technology, 2007, 15(3): 343-346.

[10] VAPNIK V N. The nature of statistical learning theory $[\mathrm{M}]$. N Y:Springer-Verlag, 1995.

[11] ZHANG $X$ G. Introduction to statistical learning theory and support vector machin $[\mathrm{J}]$. Acta Automatica Sinica, 2000, 26(1):32-42.

[12] LIANG W F, WANG X D, LIANG P ER. Pressure sensor temperature compensation based on least squares support vector machine $[\mathrm{J}]$.Chinese Journal of Scientific Instrument, 2007, 28( 12) : 2235-2238.

[13] YANG Q, TIAN F, WANG D ZH, et al. Real-time fault diagnosis approach based on lifting wavelet and recursive LSSVM $[\mathrm{J}]$. Chinese Journal of Scientific Instrument, 2011, 32( 3) : $596-602$ 